\title{
EFEITOS DA IMERSÃO EM ÁGUA FRIA NA RECUPERAÇÃO DE ÍNDICES ESPECTRAIS DA VARIABILIDADE DA FREQUÊNCIA CARDÍACA PÓS-EXERCÍCIO
}

Allysiê Priscilla de Souza Cavina, Aline Castilho de Almeida, Jessica Kirsch Micheletti, Aryane Flauzino Machado, Franciele Marques Vanderlei, Jayme Netto Junior, Carlos Marcelo Pastre.

Universidade Estadual Paulista - UNESP, Faculdade de Ciência e Tecnologia - FCT, Departamento de Fisioterapia, Presidente Prudente, SP. e-mail: Iysi_cavina@hotmail.com

\section{RESUMO}

A imersão em água fria (IAF) aparece no âmbito esportivo como estratégia para recuperar danos causados pelo estresse físico do exercício. Assim, analisar desfechos na aplicação desta técnica e suas variáveis sobre o efeito da Variabilidade da Frequência Cardíaca (VFC) parece pertinente. A metodologia do estudo consistiu no total de 100 participantes alocados em cinco grupos: GC: grupo controle; e grupos de intervenção por meio de imersão em água fria (IAF): $\mathrm{G} 1: 5 \min$ à $9 \pm 1^{\circ} \mathrm{C} ; \mathrm{G} 2: 5 \min$ à $14 \pm 1^{\circ} \mathrm{C}$; $\mathrm{G} 3$ : $15 \min$ à $9 \pm 1^{\circ} \mathrm{C}, \mathrm{G} 4: 15 \mathrm{~min}$ à $14 \pm 1^{\circ} \mathrm{C}$. Após a captação da VFC basal, os participantes realizaram um protocolo de saltos e um teste de Wingate, seguidos da IAF. No índice VLF houve diferença estatística entre momentos de até 17 e 20 min e nos momentos T17 e T20 entre os grupos. Nos índices LF e HF houve diferença estatisticamente significante nos momentos de até 17, 20 e 40 min após exercício e entre os grupos no momento T20. Conclui-se que a IAF na avaliação da variabilidade global antecipa a recuperação entre 10 e 20 min comparado ao grupo controle.

Palavras-chave: Recuperação de função fisiológica, sistema nervoso autônomo, crioterapia, imersão.

\section{EFFECTS OF COLD WATER IMMERSION ON SPECTRAL INDICES OF HEART RATE VARIABILITY RECOVERY POST EXERCISE}

\begin{abstract}
Cold water immersion (CWI) appears in the sport as a strategy to recover damages caused by the physical stress of the exercise. Thus, to analyze the effects of this technique and its variables on the effect of Heart Rate Variability (HRV) seems pertinent. The study methodology consisted of a total of 100 participants allocated in five groups: CG: control group; and CWI intervention groups: $\mathrm{G} 1: 5 \mathrm{~min}$ at $9 \pm 1^{\circ} \mathrm{C}$; $\mathrm{G} 2$ : $5 \mathrm{~min}$ at $14 \pm 1^{\circ} \mathrm{C} ; \mathrm{G} 3: 15 \mathrm{~min}$ at $9 \pm 1^{\circ} \mathrm{C}, \mathrm{G} 4: 15 \mathrm{~min}$ at $14 \pm 1^{\circ} \mathrm{C}$. After baseline HRV uptake, participants performed a jump protocol and a Wingate test, followed by the CWI. In the VLF index there was a statistical difference between moments of up to 17 and 20 min and in moments T17 and T20 between groups. In the LF and HF indices there was a statistically significant difference in the moments of up to 17, 20 and 40 min after exercise and between the groups at time T20. It is concluded that CWI in the assessment of global variability anticipates recovery between 10 and 20 min compared to the control group.
\end{abstract}

Keywords: Recovery of function, autonomic nervous system, cryotherapy, immersion.

\section{INTRODUÇÃO}

Técnicas de recuperação pós-exercício são temas amplamente discutidos na pesquisa cientifica, sendo de vasta importância para o domínio do esporte. Tais técnicas visam reparar o estresse causado pelo exercício melhorando assim o rendimento de atletas de alta performance ${ }^{1}$.

Uma das técnicas investigadas é a Imersão em Água Fria (IAF), ferramenta de fácil acesso e baixo custo bastante utilizada entre os profissionais em campo ${ }^{1}$.

Sabe-se que a IAF promove uma analgesia local, e auxilia para diminuição de concentração de fluidos, diminuindo o edema e respostas inflamatórias agudas decorrentes do estresse muscular, através da vasoconstrição localizada ${ }^{2,3}$.

Pode-se citar como efeito da IAF a pressão hidrostática, que é eficaz em aumentar o débito cardíaco, seguido de um aumento da 
circulação sanguínea muscular removendo assim os metabólicos produzidos durante a execução do exercício de volta para o sangue ${ }^{4}$. Outro efeito da técnica é a vasoconstrição que leva a um redirecionamento do fluxo de sangue das vias periféricas, ocasionando assim um aumento da força cardíaca e diminuindo o esforço cardiovascular. Isso é observado por meio de frequências cardíacas mais baixas, analisadas durante a recuperação e após IAF, o que aumenta a percepção de recuperação após o exercício ${ }^{5-7}$.

Há opções clássicas utilizadas para inferir sobre a recuperação após exercícios. Entretanto, tem-se observado a análise da variabilidade da frequência cardíaca (VFC) e seus índices como indicadores de recuperação do sistema nervoso autônomo (SNA) $)^{1,4}$.

A modulação autonômica cardíaca é medida pela análise VFC, que além de ser uma ferramenta de fácil acesso, ela avalia a função autonômica do coração de maneira não invasiva por meio de series temporais dos intervalos RR (entre um batimento e outro) obtidos através do sinal eletrocardiográfico, sendo que suas variações viabilizam informações do SNA e seu efeito no coração $0^{9,10}$.

De acordo com os estudos de Parouty et al. ${ }^{11}$ a IAF foi associada a uma maior atividade parassimpática pré esforço, uma diminuição da frequência cardíaca o que ocasionou mudanças nos tempos entre os sprints avaliados. Corroborando Al Haddad et al. ${ }^{7}$ observaram, que independente da temperatura, a imersão favorece o sistema parassimpático resultando valores de RMSSD (raiz quadrada da somatória do quadrado das diferenças entre os intervalos RR no registro $)^{10}$ maiores, assim como Bastos et al. ${ }^{12}$ que observaram efeito positivo da técnica nos índices RMSSD e HF, em comparação às demais técnicas recuperativas, sendo a mesma realizada a $11 \pm 2^{\circ} \mathrm{C}$ por 6 minutos. Os autores ${ }^{12}$ no entanto, discutem que tais diferenças ao fim de um período maior não são significantes. Por outro lado, Halson et al. ${ }^{13}$ em seu estudo relatam que temperaturas abaixo de $5^{\circ} \mathrm{C}$ não são adequadas para a recuperação pós-exercício.

Bleakley et al. ${ }^{14}$ afirmam que estudos de alta qualidade neste meio devem ser incentivados para responder a questões diversas, sobretudo considerando amostras maiores e ainda seus efeitos adversos. Dessa forma, quando observados os diversos estudos que utilizam a IAF como técnica de recuperação pós-exercício, notam-se divergências quanto às estratégias de aplicação de técnicas ou mesmo aos modelos de estresse utilizados em cada estudo.

Portanto, algumas lacunas ainda merecem ser preenchidas para um melhor entendimento do processo, como: quais os melhores tempos e temperaturas para aplicação da IAF em índices da VFC. Embora sejam consideradas as mudanças nos índices de VFC nos achados, não se faz menção da análise de diferentes tempos e temperaturas de aplicação, sobretudo com ênfase nos índices espectrais da VFC. Assim, assumindo que a IAF pode promover efeitos positivos sobre os índices de VFC, entende-se como pertinente testar variações de tempo e temperatura de aplicação sobre os mesmos.

O objetivo desse estudo foi analisar o efeito da IAF como técnica de recuperação após exercício exaustivo a partir de diferentes temperaturas e tempos de aplicação sobre os índices lineares espectrais.

\section{METODOLOGIA}

Casuística

Inicialmente realizou-se um cálculo para obtenção do tamanho amostral baseando-se em dados prévios do estudo de Bastos et al. ${ }^{12}$ a partir dos valores de SDNN (representa o desvio-padrão da média de todos os intervalos RR normais, expresso em $\mathrm{mseg}^{10}$ dos participantes em repouso com $57 \pm 20,5$ mseg e uma diferença mínima de 18 msef para esta média, no qual deve-se alocar 20 participantes por grupo considerando o teste de hipótese bicaudal, $80 \%$ de poder do teste e $5 \%$ de significância estatística. Foram considerados critérios de inclusão para formação dos grupos, tais como: ausência de anemia, processo inflamatório, diabetes, doença cardiovascular, problemas no fígado, não etilistas, não fumantes, não ser usuários de drogas ilícitas ${ }^{15}$.

\section{Declaração de Ética e inclusão no estudo}

Participantes foram informados sobre os procedimentos e objetivos do estudo, e após concordarem, assinaram um termo de consentimento livre e esclarecido, onde ficou assegurada a privacidade dos mesmos. 0 presente estudo foi aprovado pelo Comitê de Ética em Pesquisa da Faculdade de Ciências e Tecnologia - FCT/UNESP, sob número de protocolo 51903.

Grupos experimentais 
O estudo foi composto por cinco grupos, sendo um o controle $(G C)$, que não sofreu intervenção recuperativa, e outros quatros grupos que sofreram intervenção (G1, G2, G3 e G4). Dessa forma, 100 participantes foram alocados nos mesmos.

\section{Delineamento do ensaio}

Anteriormente às coletas de dados, os participantes foram orientados a não realizar exercícios físicos rigorosos por 24 h, não consumir bebidas alcóolicas e/ou estimulantes tais como café, chá e refrigerantes a base de cola, realizar uma refeição leve pelo menos duas horas antes dos procedimentos, bem como portar roupas e calçados confortáveis.

A coleta de dados foi realizada no Laboratório de Fisioterapia Desportiva (LAFIDE) da Faculdade de Ciências e Tecnologia FCT/UNESP, respeitando o horário das $17 \mathrm{~h} 30$ às $22 \mathrm{~h} 00$. Todos os protocolos foram realizados conforme experimentos já realizados de mesma natureza em um laboratório sob condições padronizadas (temperatura: $22 \pm 1^{\circ} \mathrm{C}$, umidade relativa: $40-60 \%)^{17}$.

Posteriormente à coleta de dados antropométricos, posicionou-se no tórax dos participantes a cinta de captação e no seu punho o receptor de FC. Simultaneamente à ativação do monitor de FC, acionou-se um cronômetro para facilitar o registro dos tempos de cada etapa do protocolo experimental. A captação da VFC basal aconteceu por 20 minutos.

\section{Protocolo de estresse}

Após a captação da VFC basal, todos os participantes realizaram um protocolo de saltos composto por 10 séries de 10 saltos com um minuto de intervalo entre as séries. Durante a realização dos saltos, os participantes foram orientados a posicionar as mãos no quadril e durante o pouso a flexionar os joelhos a 90 graus a fim de evitar compensações ${ }^{18}$.

Poteriormente, os participantes realizaram o protocolo do Teste Anaeróbico de Wingate, que foi realizado num cicloergômetro (CG04 Inbramed, Porto Alegre, Brasil). Realizouse um aquecimento de $5 \mathrm{~min}$, pedalando de 60 a $90 \mathrm{rpm}$ com uma carga fixa de $1,0 \mathrm{Kg}$, com sprints no 2 - e 4 o min e o teste propriamente dito, que requer o máximo de pedaladas em $30 \mathrm{seg} \mathrm{com}$ carga estipulada de $0,075 \mathrm{kp} \cdot \mathrm{kg}^{-1}$ da massa corporal do participante.
Protocolos de Recuperação

Imediatamente após a o protocolo de estresse, os participantes dos grupos de intervenção foram imersos até a altura da espinha ilíaca ântero-superior em um tanque com água fria no qual ficaram sentados em um banco para o procedimento de recuperação ${ }^{12}$ a partir de diferentes tempos de exposição e temperaturas da água (G1: 5 min à $9 \pm 1^{\circ} \mathrm{C}$; G2: 5 min à $14 \pm$ $1^{\circ} \mathrm{C}$; G3 15 min à $9 \pm 1^{\circ} \mathrm{C}$; e G4: 15 min à $14 \pm 1^{\circ} \mathrm{C}$ ). Após esse procedimento, o participante permaneceu sentado em uma cadeira durante 1 h e 15 min para a captação da VFC nos demais momentos.

Os participantes do grupo controle (GC) permaneceram sentados fora do tanque imediatamente após o protocolo de exercício, portanto, todas as captações para os momentos de análise de VFC foram realizadas nessa posição. Para G1 e G2 o momento T7 foi captado com o participante dentro no tanque e os demais momentos fora do tanque. Já para G3 e G4, os momentos T7 e T17 foram captados com o participante dentro do tanque e os demais momentos, fora do tanque. Optou-se por avaliar esses momentos para investigar os efeitos imediatos da imersão, sendo considerados 2 minutos de intervalo para o término do protocolo de recuperação e o deslocamento até a cadeira, onde permaneceu sentado até o final das análises (mais $1 \mathrm{~h}$ e $10 \mathrm{~min}$ ou $1 \mathrm{~h}$, de acordo com as características do grupo que sofreram a intervenção ou $1 \mathrm{~h}$ e $15 \mathrm{~min}$ para o grupo controle).

A VFC foi analisada no sentido de verificar a recuperação autonômica cardíaca em três domínios: tempo, frequência e caos. Os registros foram captados em seis momentos: antes do esforço (Basal), em 7 (T7), 17 (T17), 20 (T20), 40 (T40) e 60 (T60) min após a execução do exercício.

Os tempos de exposição e temperaturas de aplicação da técnica foram definidos por meio de investigação prévia realizada na literatura científica, na qual prevaleceram os tempos de 5 e 15 min e temperaturas entre 10 e $15^{\circ} \mathrm{C}$.

Análise da variabilidade da frequência cardíaca

Para determinação da VFC utilizou-se um cardiofrequencímetro da marca Polar Electro Oy - modelo RS800CX, já utilizado em outro estudo $^{12}$. Os dados obtidos por meio da monitorização foram removidos do software Polar Pro Trainer, versão 5.0 e posteriormente analisados pelo Kubios HRV Analysis Software 1.1 
for Windows (The Biomedical Signal Analysis Group, Department of Applied Physics, University of Kuopio, Finland) ${ }^{16}$. A filtragem do sinal foi realizada de forma manual, caracterizada pela inspeção visual dos intervalos R-R e exclusão de intervalos anormais, por meio do registro de 256 (momentos T7 e T17) e 1000 (demais momentos) intervalos entre os batimentos cardíacos em cada período de análise.

Foram utilizados para análise os índices lineares do domínio da frequência: 1) Componente de muito baixa frequência (very low frequency: VLF) com variação menor que $0,04 \mathrm{~Hz}$; 2) Componente de baixa frequência (low frequency: LF), com variação entre 0,04 e $0,15 \mathrm{~Hz}$; 3) Componente de alta frequência (high frequency: $\mathrm{HF}$ ), com variação de 0,15 a $0,4 \mathrm{~Hz}^{10}$. A análise espectral foi calculada utilizando 0 algoritmo da transformada rápida de Fourier.

Análise dos resultados

Inicialmente os grupos foram comparados segundo variáveis antropométricas e idade, visando à verificação da homogeneidade dos participantes da pesquisa. A distribuição quanto à normalidade dos dados foi testada por meio do teste de Kolmogorov-Smirnov. Dessa forma, a distribuição desses dados foi considerada normal, sendo, portanto utilizada a análise paramétrica e realizado a Análise de variâncias one-way (ANOVA), complementado pelo teste de Tukey, com $p>0,05$.
Para a comparação dos índices de VFC entre os diferentes protocolos (GC vs $G 1$ vs $G 2$ vs G3 vs G4) e momentos (basal vs T7, T17, T20, T40 e T60) foi feita por meio da técnica de análise de variância para modelo de medidas repetidas (ANOVA) no esquema de dois fatores. Como a distribuição dos dados de todos os índices foram considerados não normais, também por meio do teste de Kolmogorov-Smirnov, a análise foi complementada com o teste de Dunn para dados não paramétricos. Para análise da interação dos dados, primeiramente o mesmo foram checados quanto à violação de esfericidade utilizando-se o teste de Mauchly's e a correção de GreenhouseGeisser foi utilizada quando a esfericidade foi violada. Considerou-se o nível de significância em $p<0,05$ para todos os testes.

\section{RESULTADOS}

Para o índice VLF (Tabela 1) observou-se diferença estatisticamente significante na comparação entre os momentos em até 20 min após o exercício em relação ao momento basal para GC, G1 e G2 e em até 17 min para G3 e G4. $\mathrm{Na}$ comparação entre os grupos, observou-se diferença nos momentos T17 no G4 em relação ao GC e em T20 no G3 e G4 também em relação ao $\mathrm{GC}$.

Tabela 1. Medianas, valores mínimos e máximos de VLF, de acordo com os diferentes grupos e momentos de análise.

\begin{tabular}{|c|c|c|c|c|c|c|}
\hline \multirow[b]{2}{*}{ Grupos } & \multicolumn{6}{|c|}{ Momentos de análise } \\
\hline & Basal & T7 & T17 & T20 & T40 & T60 \\
\hline $\begin{array}{l}\text { Controle } \\
(n=21)\end{array}$ & $\begin{array}{c}1127 \\
(441 ; 4875)\end{array}$ & $\begin{array}{c}101 \\
(33 ; 7853)^{*}\end{array}$ & $\begin{array}{c}81 \\
(9 ; 1311)^{*}\end{array}$ & $\begin{array}{c}267 \\
(56 ; 2198)^{*}\end{array}$ & $\begin{array}{c}531 \\
(120 ; 7353)\end{array}$ & $\begin{array}{c}829 \\
(114 ; 10317)\end{array}$ \\
\hline $\begin{array}{c}\mathbf{G 1} \\
(n=16)\end{array}$ & $\begin{array}{c}1717 \\
(363 ; 4864)\end{array}$ & $\begin{array}{c}162 \\
(18 ; 1573)^{*}\end{array}$ & $\begin{array}{c}116 \\
(33 ; 1777)^{*}\end{array}$ & $\begin{array}{c}316 \\
(58 ; 1139)^{*}\end{array}$ & $\begin{array}{c}706 \\
(218 ; 2919)\end{array}$ & $\begin{array}{c}2013 \\
(503 ; 5218)\end{array}$ \\
\hline $\begin{array}{c}\text { G2 } \\
(n=16)\end{array}$ & $\begin{array}{c}1325 \\
(331 ; 3792)\end{array}$ & $\begin{array}{c}397 \\
(22 ; 1748)^{*}\end{array}$ & $\begin{array}{c}262 \\
(44 ; 1264)^{*}\end{array}$ & $\begin{array}{c}535 \\
(110 ; 1307)^{*}\end{array}$ & $\begin{array}{c}1118 \\
(279 ; 2727)\end{array}$ & $\begin{array}{c}1748 \\
(458 ; 3075)\end{array}$ \\
\hline $\begin{array}{c}\text { G3 } \\
(n=15)\end{array}$ & $\begin{array}{c}904 \\
(328 ; 5146)\end{array}$ & $\begin{array}{c}73 \\
(20 ; 291)^{*}\end{array}$ & $\begin{array}{c}444 \\
(50 ; 4323)^{*}\end{array}$ & $\begin{array}{c}1275 \\
(484 ; 3943) \#\end{array}$ & $\begin{array}{c}786 \\
(181 ; 2744)\end{array}$ & $\begin{array}{c}887 \\
(450 ; 3407)\end{array}$ \\
\hline $\begin{array}{c}\mathbf{G 4} \\
(n=16)\end{array}$ & $\begin{array}{c}1934 \\
(160 ; 4221)\end{array}$ & $\begin{array}{c}72 \\
(8 ; 541)^{*}\end{array}$ & $\begin{array}{c}877 \\
(25 ; 6240)^{* \#}\end{array}$ & $\begin{array}{c}2375 \\
(352 ; 4773) \#\end{array}$ & $\begin{array}{c}873 \\
(143 ; 5002)\end{array}$ & $\begin{array}{c}2076 \\
(172 ; 9253)\end{array}$ \\
\hline
\end{tabular}

${ }^{*} p<0,05$ na comparação entre os tempos (momentos T7, T17, T20, T40 e T60) em relação ao momento basal. \# $p<0,05$ na comparação entre os grupos em relação ao GC.

GC: grupo controle, G1: $5 \min$ à $9 \pm 1^{\circ} \mathrm{C}, \mathrm{G} 2: 5 \min$ à $14 \pm 1^{\circ} \mathrm{C}, \mathrm{G} 315 \min$ à $9 \pm 1^{\circ} \mathrm{C}$, e G4: $15 \min$ à $14 \pm 1^{\circ} \mathrm{C}$. 
Para o índice LF (Tabela 2), observou-se diferença estatisticamente significante na comparação entre os momentos em até $20 \mathrm{~min}$ após o exercício em relação ao momento basal para GC, G1, G2 e G4 em até 17 min para G3. Na comparação entre os grupos, observou-se diferença estatisticamente significante no momento T20 para G3 e G4 em relação ao GC.
Para o índice HF (tabela 3), observou-se diferença estatisticamente significante na comparação entre os momentos em até 40 minutos após o exercício em relação ao momento basal para GC e em até 20 minutos para os demais grupos. Na comparação entre os grupos, não foram observadas diferenças estatisticamente significantes.

Tabela 2. Medianas, valores mínimos e máximos de LF, de acordo com os diferentes grupos e momentos de análise.

\begin{tabular}{|c|c|c|c|c|c|c|}
\hline \multirow[b]{2}{*}{ Grupos } & \multicolumn{6}{|c|}{ Momentos de análise } \\
\hline & Basal & T7 & T17 & T20 & T40 & T60 \\
\hline $\begin{array}{c}\text { Controle } \\
(n=21)\end{array}$ & $\begin{array}{c}1220 \\
(294 ; 3885)\end{array}$ & $\begin{array}{c}44 \\
(5 ; 390)^{*}\end{array}$ & $\begin{array}{c}103 \\
(15 ; 223)^{*}\end{array}$ & $\begin{array}{c}121 \\
(16 ; 865)^{*}\end{array}$ & $\begin{array}{c}557 \\
(72 ; 2651)\end{array}$ & $\begin{array}{c}1123 \\
(99 ; 2336)\end{array}$ \\
\hline $\begin{array}{c}\mathbf{G 1} \\
(\mathrm{n}=16)\end{array}$ & $\begin{array}{c}1689 \\
(246 ; 2889)\end{array}$ & $\begin{array}{c}30 \\
(6 ; 175)^{*}\end{array}$ & $\begin{array}{c}74 \\
(9 ; 572)^{*}\end{array}$ & $\begin{array}{c}153 \\
(14 ; 1027)^{*}\end{array}$ & $\begin{array}{c}864 \\
(80 ; 1986)\end{array}$ & $\begin{array}{c}978 \\
(296 ; 2860)\end{array}$ \\
\hline $\begin{array}{c}\mathbf{G 2} \\
(\mathrm{n}=16)\end{array}$ & $\begin{array}{c}1728 \\
(88 ; 4513)\end{array}$ & $\begin{array}{c}63 \\
(8 ; 156)^{*}\end{array}$ & $\begin{array}{c}146 \\
(34 ; 585)^{*}\end{array}$ & $\begin{array}{c}240 \\
(37 ; 1454)^{*}\end{array}$ & $\begin{array}{c}750 \\
(183 ; 3717)\end{array}$ & $\begin{array}{c}1433 \\
(249 ; 4046)\end{array}$ \\
\hline$\underset{(n=15)}{\mathbf{G 3}}$ & $\begin{array}{c}1000 \\
(413 ; 3444)\end{array}$ & $\begin{array}{c}27 \\
(7 ; 413)^{*}\end{array}$ & $\begin{array}{c}109 \\
(18 ; 433)^{*}\end{array}$ & $\begin{array}{c}305 \\
(50 ; 1626) \#\end{array}$ & $\begin{array}{c}901 \\
(146 ; 2586)\end{array}$ & $\begin{array}{c}903 \\
(267 ; 2537)\end{array}$ \\
\hline $\begin{array}{c}\mathbf{G 4} \\
(n=16)\end{array}$ & $\begin{array}{c}1420 \\
(134 ; 2836)\end{array}$ & $\begin{array}{c}25 \\
(8 ; 122)^{*}\end{array}$ & $\begin{array}{c}86 \\
(24 ; 1013)^{*}\end{array}$ & $\begin{array}{c}255 \\
(45 ; 1966) * \#\end{array}$ & $\begin{array}{c}975 \\
(177 ; 3041)\end{array}$ & $\begin{array}{c}1551 \\
(283 ; 336)\end{array}$ \\
\hline
\end{tabular}

Tabela 3. Medianas, valores mínimos e máximos de HF, de acordo com os diferentes grupos e momentos de análise.

\begin{tabular}{|c|c|c|c|c|c|c|}
\hline \multirow[b]{2}{*}{ Grupos } & \multicolumn{6}{|c|}{ Momentos de análise } \\
\hline & Basal & T7 & T17 & T20 & $\mathrm{T} 40$ & T60 \\
\hline $\begin{array}{c}\text { Controle } \\
(n=21)\end{array}$ & $\begin{array}{c}448 \\
(51 ; 3639)\end{array}$ & $\begin{array}{c}11 \\
(1 ; 313)^{*}\end{array}$ & $\begin{array}{c}14 \\
(1 ; 83)^{*}\end{array}$ & $\begin{array}{c}17 \\
(2 ; 147)^{*}\end{array}$ & $\begin{array}{c}88 \\
(5 ; 804)^{*}\end{array}$ & $\begin{array}{c}188 \\
(5 ; 1805)\end{array}$ \\
\hline $\begin{array}{c}\mathbf{G 1} \\
(\mathrm{n}=16)\end{array}$ & $\begin{array}{c}557 \\
(126 ; 1643)\end{array}$ & $\begin{array}{c}11 \\
(1 ; 234)^{*}\end{array}$ & $\begin{array}{c}5 \\
(1 ; 35)^{*}\end{array}$ & $\begin{array}{c}19 \\
(3 ; 190)^{*}\end{array}$ & $\begin{array}{c}171 \\
(18 ; 973)\end{array}$ & $\begin{array}{c}327 \\
(76 ; 1460)\end{array}$ \\
\hline $\begin{array}{c}\mathbf{G 2} \\
(\mathrm{n}=16)\end{array}$ & $\begin{array}{c}622 \\
(25 ; 4910)\end{array}$ & $\begin{array}{c}18 \\
(2 ; 110)^{*}\end{array}$ & $\begin{array}{c}5 \\
(1 ; 33)^{*}\end{array}$ & $\begin{array}{c}31 \\
(2 ; 285)^{*}\end{array}$ & $\begin{array}{c}122 \\
(25 ; 1047)\end{array}$ & $\begin{array}{c}283 \\
(59 ; 2243)\end{array}$ \\
\hline $\begin{array}{c}\mathbf{G 3} \\
(n=15)\end{array}$ & $\begin{array}{c}458 \\
(92 ; 1535)\end{array}$ & $\begin{array}{c}20 \\
(3 ; 99)^{*}\end{array}$ & $\begin{array}{c}5 \\
(1 ; 24)^{*}\end{array}$ & $\begin{array}{c}41 \\
(5 ; 200)^{*}\end{array}$ & $\begin{array}{c}216 \\
(31 ; 515)\end{array}$ & $\begin{array}{c}246 \\
(34 ; 782)\end{array}$ \\
\hline $\begin{array}{c}\text { G4 } \\
(n=16)\end{array}$ & $\begin{array}{c}406 \\
(58 ; 1700)\end{array}$ & $\begin{array}{c}13 \\
(1 ; 111)^{*}\end{array}$ & $\begin{array}{c}6 \\
(1 ; 37)^{*}\end{array}$ & $\begin{array}{c}33 \\
(7 ; 331)^{*}\end{array}$ & $\begin{array}{c}151 \\
(28 ; 1697)\end{array}$ & $\begin{array}{c}309 \\
(52 ; 2770)\end{array}$ \\
\hline
\end{tabular}

* $p<0,05$ na comparação entre os tempos (momentos T7, T17, T20, T40 e T60) em relação ao momento basal. \# p<0,05 na comparação entre os grupos em relação ao GC.

GC: grupo controle, G1: $5 \min$ à $9 \pm 1^{\circ} \mathrm{C}, \mathrm{G} 2: 5 \min$ à $14 \pm 1^{\circ} \mathrm{C}, \mathrm{G} 315 \min$ à $9 \pm 1^{\circ} \mathrm{C}$, e G4: $15 \min$ à $14 \pm 1^{\circ} \mathrm{C}$. 


\section{DISCUSSÃO}

A partir das análises dos dados pode-se observar que, considerando o retorno dos valores de recuperação a seus níveis basais observa-se que $60 \mathrm{~min}$ é tempo suficiente para quaisquer situações independente da realização ou não de intervenção para recuperação do balanço simpato-vagal e que em índices que avaliam a variabilidade global parece haver antecipação da recuperação entre 10 e $20 \mathrm{~min}$ quando da aplicação da IAF ou apenas para o grupo que realizou a imersão por maiores temperaturas (G3 e G4).

As hipóteses para os desfechos descritos estão relacionadas a dois fatores. $O$ estímulo a barorreceptores e a resposta à redução de temperatura a partir da termorregulação. Os barorreceptores, que são sensíveis às mudanças no volume sanguíneo, são estimulados pelo redirecionamento de fluxo da periferia para a região torácica e o consequente aumento no volume sanguíneo central, devido à pressão hidrostática, o que induz a redução da modulação simpática concomitante com a modulação parassimpática ${ }^{5,6}$. No caso da redução de temperatura, os termorreceptores na pele, tecidos subcutâneos e vasos sanguíneos são estimulados, o que aumenta a vasoconstrição e o reflexo parassimpático cardíaco, além de potencializar o redirecionamento de fluxo para proteção de órgãos vitais ${ }^{18-21}$

Observou-se que, maiores temperaturas de imersão parecem promover uma maior recuperação desses índices, refletida em valores estatisticamente superiores em relação ao GC. Embora tenham utilizado tempos inferiores a 15 min, a temperatura de $14^{\circ} \mathrm{C}$ também foi utilizada nos artigos.

Machado et al. ${ }^{22}$ em uma revisão sistemática e meta-análise avaliaram os efeitos da IAF pós-exercício em diferentes dosagens e identificou que a IAF pode ser melhor do que a recuperação passiva na gestão da dor muscular e também a presença de uma relação doseresposta, quando aplicada entre 11 e $15^{\circ} \mathrm{C}$ para 11-15 min. Al Haddad et al. ${ }^{7}$ enfatizaram que a IAF realizada a $14^{\circ} \mathrm{C}$ pode estimular receptores de frio e promover um efeito cumulativo de respostas hemodinâmicas através do sistema de termorregulação, favorecendo um melhor controle autonômico.

Sabe-se que o índice VLF sofre influência do sistema termorregulador ${ }^{21}$, além de representar a ação dos ramos simpático e parassimpático do $S N A^{10}$. Tais achados podem ser atribuídos à ativação cardiovagal durante a IAF que é promovida pela diminuição brusca da temperatura após a imersão, o que estimula os termorreceptores induzindo a modulação vagal, além da ação dos barorreceptores ${ }^{5,6}$. Apesar da descrição da relação entre função autonômica e o índice em questão, não foi observada sua abordagem nos artigos levantados para realização desta pesquisa.

Analisando os achados encontrados entre os índices, e considerando a representação autonômica de cada um deles, pode-se observar que os índices que representam a variabilidade global, ou seja, refletem a ação de ambos os ramos simpático e parassimpático do SNA, apresentam antecipação do momento de recuperação entre 10 e $20 \mathrm{~min}$, ou em todos os grupos que realizaram a IAF, ou no grupo que realizou a imersão por maiores tempo e temperatura. Entretanto, os índices que representam a ação parassimpática não indicam tal comportamento, ou seja, todos os grupos se recuperam no mesmo momento. Porém, de modo geral, destacam-se os melhores valores e melhor recuperação para G4 na maioria dos índices avaliados.

A aplicação da IAF promove, portanto, efeitos em ambos os ramos do SNA, de modo a inibir a modulação simpática e acelerar a modulação vagal. Concomitantemente a esta resposta, alguns estudos, ${ }^{6,23-25)}$ mostram que ocorre uma cascata de respostas promovidas pelo sistema hemodinâmico, incluindo redirecionamento de fluxo da periferia para regiões centrais do tórax, aumentando o retorno venoso e o débito cardíaco, contribuindo para um maior balanço simpatovagal.

Além disso, observa-se que a temperatura e o tempo de imersão podem ser fatores determinantes para otimizar as respostas do SNA, podendo-se inferir que, quando o foco é a recuperação do balanço simpato-vagal, as melhores opções de técnicas de IAF são com temperaturas de $14^{\circ} \mathrm{C}$.

Como limitações do estudo pode-se apontar que, embora todos os participantes deste estudo tenham sido classificados como fisicamente ativos, a partir do questionário de atividade física (IPAQ), não foi possível garantir a especificidade das atividades que os mesmos realizavam, caracterizando uma limitação, visto que o nível e o tipo de atividade física praticada exercem influência sobre o SNA. Além disso, 
pode-se apontar a não investigação dos parâmetros cardiorrespiratórios, como pressão arterial, frequência cardíaca e frequência respiratória. Tais parâmetros poderiam inferir se essas estratégias também poderiam influenciálos positivamente, ou quais seriam as mais adequadas para a recuperação deste sistema e se poderiam ser influenciadas pelas respostas obtidas pela modulação autonômica cardíaca.

\section{CONCLUSÃO}

A partir dos achados do presente estudo, pode-se concluir que a IAF nos índices que avaliam a variabilidade global VLF e LF parece haver antecipação da recuperação entre 10 e 20 min nos grupos que realizaram a IAF e no índice que representa a ação parassimpática não houve diferenças nos momentos de recuperação, porém G3 e G4 apresenta os melhores valores. Portanto, caso o objetivo do processo de recuperação aborde a restauração da modulação autonômica cardíaca, sugere-se a técnica com ênfase em temperaturas próximas a $14^{\circ} \mathrm{C}$.

\section{CONFLITO DE INTERESSES}

Os autores declaram não haver qualquer potencial conflito de interesses que possa interferir na imparcialidade deste trabalho científico.

\section{REFERÊNCIAS}

1. Pastre CM, Bastos FN, Netto Junior J, Vanderlei LCM, Hoshi RA. Métodos de Recuperação Pósexercício: uma Revisão Sistemática. Rev Bras Med Esporte. 2009;15(2):138-44. DOI: http://dx.doi.org/10.1590/S1517-

\section{2}

2. George PE et al. Effects of Water Immersion on Posttraining Recovery in Australian Footballers. Int J Sports Physiol Perform. 2012;7:357-66. DOI: http://dx.doi.org/10.1123/ijspp.7.4.357

3. Eston R, Peters D. Effects of cold water immersion on the symptoms of exercise-induced muscle damage.

J Sports Sci. 1999;17:231-8. DOI: http://dx.doi.org/10.1080/026404199366136

4. Versey NG, Halson SL, Dawson BT. Water immersion recovery for athletes: effect on exercise performance and practical recommendations. Sports Med. 2013;43(11):1101-30.

DOI:

http://dx.doi.org/10.1007/s40279-013-0063-8

5. Stanley J, Peake JM, Coombes JS, Buchheit M. Central and peripheral adjustments during highintensity exercise following cold water immersion. Eur J Appl Physiol. 2014;114:147-63. DOI: http://dx.doi.org/10.1007/s00421-013-2755-z

6. Stanley J, Buchheit M, Peake JM. The effect of postexercise hydrotherapy on subsequent exercise performance and heart rate variability. Eur J Appl Physiol. 2012;112(3):951-61. DOI: http://dx.doi.org/10.1007/s00421-011-2052-7

7. Al Haddad $H$, Laursen $P B$, Chollet $D$, Lemaitre $F$, Ahmaidi S, Buchheit $M$. Effect of cold or thermoneutral water immersion on post-exercise heart rate recovery and heart rate variability indices. Auton Neurosci. 2010;156:111-6. DOI: https://dx.doi.org/10.1016/i.autneu.2010.03.017

8. Bailey DM, Erith SJ, Griffin PJ, Dowson A, Brewer DS, Williams NGC. Influence of cold-water immersion on indices of muscle damage following prolonged intermittent shuttle running. J Sports Sci. 2007; 25(11):1163-1170.

DOI:

http://dx.doi.org/10.1080/02640410600982659

9. Pumprla J, Howorka K, Groves D, Chester M, Nolan J. Functional assessment of heart rate variability: physiological basis and practical applications. Int J Cardiol. 2002;84:1-14. DOI: http://dx.doi.org/10.1016/S0167-5273(02)00057-8

10. VANDERLEI, Luiz Carlos Marques et al . Noções básicas de variabilidade da frequência cardíaca e sua aplicabilidade clínica. Rev Bras Cir Cardiovasc (São José do Rio Preto). 2009;24(2):205-17.

11. Parouty J, Al Haddad H, Quod M, Leprêtre PM, Ahmaidi S, Buchheit M. Effect of cold water immersion on 100-m sprint performance in well-trained swimmers. Eur J Appl Physiol. 2010;109(3):483-90. DOI: http://dx.doi.org/10.1007/s00421-010-1381-2

12. Bastos FN, Vanderlei LCM, Nakamura FY, Bertollo M, Hoshi RA, Netto Júnior J; Godoy MF, Pastre CM. Effects of cold water immersion and active recovery on post-exercise heart rate variability. Int J Sports Med. 2012;33(11):873-9. DOI: http://dx.doi.org/10.1055/s-0032-1301905

13. Halson SL, Quod MJ, Martin DT, Gardner AS, Ebert TR, Laursen PB. Physiological responses to cold water immersion following cycling in the heat. Int J Sports Physiol Perform. 2008;3(3):331-46. DOI: http://dx.doi.org/10.1123/ijspp.3.3.331

14. Bleakley C, McDonough S, Gardner E, Baxter GD, Hopkins JT, Davison GW. Cold-water immersion (cryotherapy) for preventing and treating muscle soreness after exercise (Review). Cochrane Database Syst Rev 2012; issue 2.

15. Twist C, Eston R. The effects of exercise-induced muscle damage on maximal intensity intermittent exercise performance. Eur J appl Physiol. 2005;94:6528. DOI: http://dx.doi.org/10.1007/s00421-005-1357-9 16. Niskanen JP, Tarvainen MP, Ranta-Aho PO, Karjalainen PA. Software for advanced HRV analysis. Comput Methods Programs Biomed. 2004;76(1):7381.

DOI: http:/dx./doi.org/10.1016/i.cmpb.2004.03.004 17. Ascensão A, Leite $M$, Rebelo A N, Magalhaes $S$, Magalhaes J. Effects of cold water immersion on the recovery of physical performance and muscle damage following a one-off soccer match. J Sports Sci. 2011;29:217-25. DOI: http://dx.doi.org/10.1080/02640414.2010.526132 
18. Acharya UR, Faust O, Sree V, Swapna G, Martis RJ, Kadrib, NA, Suri JS. Linear and nonlinear analysis of normal and CAD-affected heart rate signals. Comput Methods Programs Biomed. 2014;113(1):55-68. DOI: http://dx.doi.org/10.1016/i.cmpb.2013.08.017

19. Vaile J, O'Hagan C, Stefanovic B, Walker M, Gill N, Askew CD. Effect of cold water immersion on repeated cycling performance and limb blood flow. Br J Sports Med. 2011;45(10):825-9. DOI: http://dx.doi.org/10.1136/bjsm.2009.067272

20. Al Haddad H, Laursen PB, Ahmaidi S, Buchheit M. Influence of cold water face immersion on postexercise parasympathetic reactivation. Eur J Appl Physiol. 2010;108:599-606. DOI: http://dx.doi.org/10.1007/s00421-009-1253-9

21. Mourot L, Bouhaddi M, Gandelin E, Cappelle S, Dumoulin G, Wolf JP, Rouillon JD, Regnard J. Cardiovascular autonomic control during short-term thermoneutral and cool head-out immersion. Aviat Space Environ Med. 2008;79(1):14-20. DOI: http://dx.doi.org/10.3357/ASEM.2147.2008

22. Machado AF et al. Can Water Temperature and Immersion Time Influence the Effect of Cold Water Immersion on Muscle Soreness? A Systematic Review and Meta-Analysis. Sports Med. 2016;46(4):503-14. DOI: http://dx.doi.org/10.1007/s40279-015-0431-7

23. Tripathi KK. Very low frequency oscillations in the power spectra of heart rate variability during dry supine immersion and exposure to non-hypoxic hypobaria. Physiol Meas. 2011;32:717-29. DOI: http://dx.doi.org/10.1088/0967-3334/32/6/008

24. Buchheit $M$, Peiffer JJ, Abbiss CR, Laursen PB. Effect of cold water immersion on postexercise parasympathetic reactivation. Am J Physiol Heart Circ Physiol. 2009;296(2):421-27. DOI: http://dx.doi.org/10.1152/ajpheart.01017.2008

25. Bleakley C, McDonough S, Gardner E, Baxter GD, Hopkins JT, Davison GW. Cold-water immersion (cryotherapy) for preventing and treating muscle soreness after exercise (Review). Cochrane Database Syst Rev 2012; issue 2.

Recebido para publicação em 19/08/2016

Revisado em 22/08/2016

Aceito em 30/08/2016 